\title{
Improved Water-Filling Power Allocation for Energy-Efficient Massive MIMO Downlink Transmissions
}

\author{
Noor Shahida M. K, Rosdiadee Nordin and Mahamod Ismail
}

\begin{abstract}
Energy Efficiency (EE) is becoming increasingly important for wireless communications and has caught more attention due to steadily rising energy costs and environmental concerns. Recently, a new network architecture known as Massive Multiple-Input Multiple-Output (MIMO) has been proposed with the remarkable potential to achieve huge gains in EE with simple linear processing. In this paper, a power allocation algorithm is proposed for EE to achieve the optimal EE in Massive MIMO. Based on the simplified expression, we develop a new algorithm to compute the optimal power allocation algorithm and it has been compared with the existing scheme from the previous literature. An improved water filling algorithm is proposed and embedded in the power allocation algorithm to maximize $\mathrm{EE}$ and Spectral Efficiency (SE). The numerical analysis of the simulation results indicates an improvement of $40 \%$ in $\mathrm{EE}$ and $50 \%$ in $\mathrm{SE}$ at the downlink transmission, compared to the other existing schemes. Furthermore, the results revealed that SE does not influence the EE enhancement after using the proposed algorithm as the number of Massive MIMO antenna at the Base Station (BS) increases.
\end{abstract}

Keywords - energy efficiency, Massive MIMO, power allocation, spectral efficiency

\section{INTRODUCTION}

$\mathbf{O}$ NE promising technology for achieving the future bandwidth requirements of the next-generation wireless communication system is Massive MIMO that has attracted lots of research attention recently [1]-[5]. Massive MIMO is based on the use of a large number of antennas to accommodate more mobile users and is allowed to improve spectrum efficiency (SE) and energy efficiency (EE) by installing massive antennas at the BS [6]. When used with suitable precoding schemes, Massive MIMO systems experience small inter-user and intercell interference, and consequently achieve significant higher throughput than the earlier multiuser-MIMO systems.

This work supported from Grant Ref. No: FRGS/1/2015/ICT04/UKM/02/2 (Malaysia's Ministry of Higher Education) and Universiti Teknikal Malaysia Melaka (UTeM)

Noor Shahida M. K is with Faculty of Electronic and Computer Engineering, Universiti Teknikal Malaysia Melaka, Hang Tuah Jaya, 76100 Durian Tunggal, Melaka, Malaysia (e-mail: noorshahida@utem.edu.my).

Rosdiadee Nordin and Mahamod Ismail are with Department of Electrical, Electronic and Systems Engineering, Faculty of Engineering and Built Environment, Universiti Kebangsaan Malaysia, 43600 UKM Bangi, Selangor, Malaysia (e-mail: \{adee,mahamod\}@ukm.edu.my).
EE has drawn lots of attention and has been considered as an important metric for evaluating a communication system, where, it focuses on maximizing EE that can reduce the total energy consumption. In existing works, there are various designs of wireless that aim for achieving energy efficient communications. In [7], the authors provide insights on the optimal design of the number of antennas, active users and transmit power for maximizing $\mathrm{EE}$ in multiuser MIMO systems, whose results show that the maximal EE is achieved by a Massive MIMO setup with hundreds of antennas deployed. Moreover, by installing a large number of antennas and with the low transmit power per-antenna, inexpensive components can be used to build the system [8].

In the past several years, valuable research results have been obtained by analyzing the EE of Massive MIMO systems. For instance, in [9] and [10], equal pilot and data power assignment for all users have been assumed in a single-cell analysis to improve the system EE. The Massive MIMO with hundreds of antennas designed in [9] has assumed perfect CSI. Resource allocation is focused in [10] to maximize EE in OFDMA systems with massive BS antennas, which takes imperfect CSI and inter-carrier interference into consideration. However, there is no power allocation scheme considered in both studies. Therefore, the authors in [11]-[16] introduced power allocation strategies to improve EE that can be described as the successfully delivered information bits per unit energy consumption. Authors [11] investigated the impact of circuit power consumption on the EE in Massive MIMO. However, the simulation results show that either the average channel gain is large or the circuit power consumption is high, the energy efficiency of the Massive MIMO system is not increasing consistently. In [12], the tradeoff between EE and SE were designed to maximize the $\mathrm{EE}$ of systems with distributed antennas. Then, the SE-EE relationship of Massive MIMO systems was investigated in [13], which showed that the EE will increase exponentially with a linear SE loss by reducing the circuit power. This result can also be interpreted as the Massive MIMO is not energy efficient to achieve very high SE because a linear increase of SE leads to an exponential decrease of EE.

The work presented in [14] developed a resource allocation of EE to maximize the EE for a multi-user OFDMA. However, an energy consumption is increasing, even though the authors in [15] showed that MIMO techniques are effective in improving capacity and SE of wireless systems. In [16], an EE power allocation has been proposed for the Massive MIMO system with Maximum Ratio Transmission (MRT) precoding and the EE and SE are improved when the number of antennas at BS 
increases and hence reduce power consumption. However, the precoding can not eliminate inter-user interference at each User Terminal (UT). Authors in [17] investigated on QoS-aware EE power allocation in downlink large-scale MIMO systems by extending the work described in [18] to overcome the problem of an EE maximization and reformulate the objective function under per-antenna transmit power and per-user rate constraints. However, the system can only achieve maximum EE when serves almost 20 users and 100 number of antennas. Recently, [19] proposed an optimal antenna selection, users and transmit power for the single-cell Massive MIMO system with zeroforcing beamforming (ZFBF), which improve the EE Massive MIMO systems. Contrary to [20], the authors focused on optimizing the transmit power at the base station for the multicell Massive MIMO system, where the maximal ratio transmission (MRT) and ZFBF with equal power allocation are used. The simulation results presented the improvement of EE by optimizing transmit power increases with the number of antennas. However, [19] and [20] optimized the total transmit power at the base station (BS), which is suboptimal for the system EE. In [21], the authors proposed a water-filling algorithm to achieve a balance between $\mathrm{EE}$ and SE with reduced complexity. However, the allocation is based on a fixed optimization model, in which each user has its water level. In this paper, we develop an EE power allocation using an improved water filling algorithm (IWF) for Massive MIMO and compare against the work reported in [16], [20] and [21] because to determine the power allocation algorithm with different power levels for the users'.

Table I

Some notations and symbols used in this paper

\begin{tabular}{|l|l|}
\hline$w_{k}$ & precoding weighting vector \\
\hline$s_{k}$ & $\begin{array}{l}\text { data symbol of the } k \text {-th (cooperatively served by } \\
\text { the sector BSs) }\end{array}$ \\
\hline$h_{k}$ & channel matrix from the $n$-th BS and the $k$-th user \\
\hline$n_{k}$ & noise vector of the $k$-th user \\
\hline$p_{k}$ & transmit power for each UT \\
\hline$r$ & distance between the user and the base station \\
\hline$B$ & spectrum bandwidth \\
\hline$R_{c}$ & distance between the user and the base station \\
\hline$\sigma^{2}$ & noise power \\
\hline$H$ & channel matrix \\
\hline$P_{\text {total }}$ & the overall power consumption \\
\hline$P_{t}$ & total transmission power consumption \\
\hline$P_{c}$ & the total of circuit power consumption \\
\hline$P_{c x}$ & power consumption at BS \\
\hline$P_{u c}$ & power consumption at UTs \\
\hline$\eta$ & power amplifier efficiency \\
\hline$R_{\min }$ & the minimum rate threshold required by user $k$ \\
\hline$g_{k}$ & the effective channel power gain to the $k$-th user \\
\hline$\mu$ & a water level \\
\hline & \\
\hline
\end{tabular}

The contributions of this paper are listed as follows: (i) both the number of antenna arrays at BS and the transmit power at the user are adjusted to maximize the EE, and (ii) we proposed a novel power allocation algorithm and compared with the another power allocation scheme from the previous works in [16], [20] and [21]. The simulation results show that it is possible to maximize EE by using the proposed power allocation compared with the work proposed in [20], [21]. The rest of the paper is organized as follows: In Section 2, the system model for the Massive MIMO system is introduced. The
EE of Massive MIMO is analyzed in Section 3. Followed by the energy efficiency power allocation scheme in Section 4. In Section 5, simulation results and analysis are reported. Finally, conclusions are presented in Section 6. For convenience, some notations and symbols used throughout the paper are listed in Table I.

\section{SYSTEM MODEL}

Consider a hexagonal cell system with the Massive MIMO systems at BS as illustrated in Fig. 1, where the systems consist of one BS with $N$ antennas and $K$ user terminals (UTs) each with $M$ antennas which are randomly and uniformly distributed in the cell with the radius $R_{c}$.

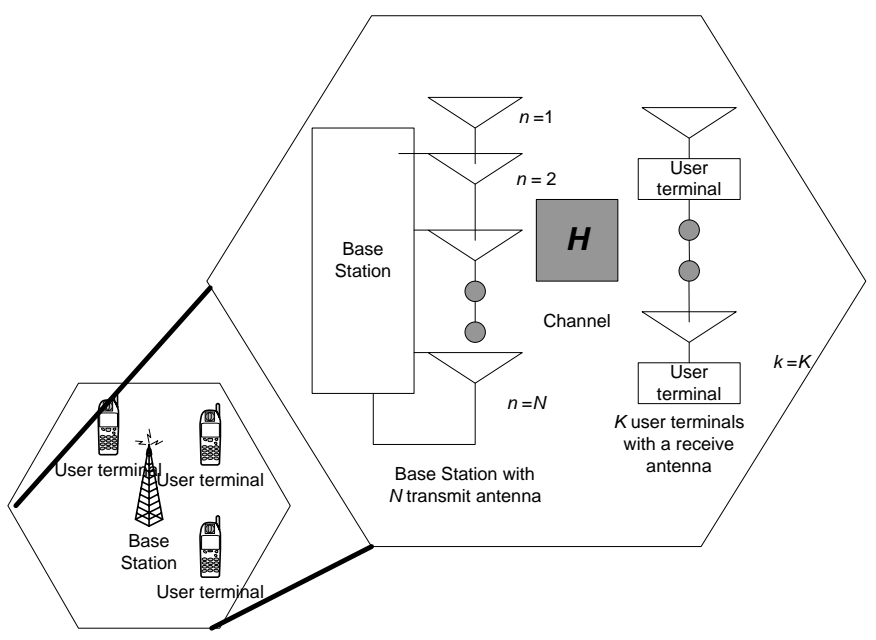

Fig. 1. Single-cell system with Massive MIMO systems at BS

Based on channel estimates, the BS forms precoding vectors to transmit data to the intended terminals. We adopt the zeroforcing $(\mathrm{ZF})$ precoding scheme in the system to reduce interuser interference. The transmitted signal vector of the system can be stated as

$$
x=W s\left[\begin{array}{lll}
w_{1,1} & \cdots & w_{1, K} \\
\vdots & \vdots \\
w_{K, 1} & \cdots & w_{K, K}
\end{array}\right]\left[\begin{array}{l}
s_{1} \\
\vdots \\
s_{K}
\end{array}\right] k=1, \ldots, K
$$

Then, the received signal of $k$-th UT can be written as

$$
y_{k}=\sqrt{p_{k}} h_{k} x_{k}+\sqrt{p_{i}} \sum_{i=1, i \neq k}^{K} h_{k} x_{i}+n_{k}
$$

$\sqrt{p_{i}} \sum_{i=1, i \neq k}^{K} h_{k} x_{i}$ indicates the existing inter-user interference.

Therefore, the signal-to-interference-plus-noise ratio (SINR) that is achieved by UT $k$ can be represented as

$$
\operatorname{SINR}_{k}=\frac{\sqrt{p_{k}}\left|h_{k} x_{k}\right|^{2}}{\sqrt{p_{i}} \sum_{i=1, i \neq k}^{K}\left|h_{k} x_{i}\right|^{2}+\sigma^{2}}
$$

Then, the achievable sum rate of the $k$-th user is

$$
R_{k}=B \sum_{k=1}^{K} \log _{2}\left(1+\operatorname{SINR}_{k}\right)
$$


Because of the simplest form, the ZF precoding is adopted at the BS and the SINR can be written as

$$
\operatorname{SINR}_{k}^{Z F}=\frac{p_{k}}{\operatorname{tr}\left(\left(H^{H} H\right)^{-1}\right)}
$$

The equation below is based on the large number of antennas at $\mathrm{BS}$ and then the theory of random matrix in [15]

$$
\frac{1}{E\left\{\operatorname{tr}\left(\left(H^{H} H\right)^{-1}\right)\right\}}=\frac{N-K}{K}, N \rightarrow \infty
$$

Then, the achievable sum rate of the system in the downlink transmission can be obtained as

$$
R_{\text {sum }}=B \log _{2}\left(1+\operatorname{SINR}_{k}\right)
$$

\section{EE FOR MASSIVE MIMO}

We define the $E E$ as

$$
E E=\frac{R_{\text {sum }}}{P_{\text {total }}}
$$

where $P_{\text {total }}$ can be written as

$$
P_{\text {total }}=\frac{1}{\eta} P_{t}+P_{c}=\frac{1}{\eta} P_{t}+\left(\sum_{k=1}^{K} P_{u c}+P_{c x}\right)=\sum_{k=1}^{K}\left(\frac{p_{k}}{\eta}+P_{u c}\right)+P_{c x}
$$

According to equation (8) and equation (9), the EE of the link can then be measured in $(\mathrm{b} / \mathrm{J} / \mathrm{Hz})$

$$
E E_{k}=\frac{R_{\text {sum }}}{P_{\text {total }}}=\frac{B \sum_{k=1}^{K} \log _{2}\left(1+\operatorname{SINR}_{k}\right)}{\sum_{k=1}^{K}\left(\frac{p_{k}}{\eta}+P_{u c}\right)+P_{c x}}
$$

\section{ENERGY EFFICIENCY POWER ALLOCATION SCHEME FOR MASSIVE MIMO}

EE is an important metric that represent transmit information bits per Joule by measuring the ratio of capacity and transmit power. Noticeably, the EE will not be very high when the transmission power is uncontrolled even though it brings much higher data rate. Hence, an appropriate transmission power allocation should be developed to achieve the best EE. In this section, the approximation of optimal power allocation is given and EE power allocation algorithm will be developed to maximize the EE. Based on equation (10), the design of an energy-efficient power allocation scheme, which includes power allocation algorithm for the multi-objective optimization problem can be summarized as:

$$
\max E E_{k}
$$

subject to

$$
\begin{gathered}
p_{k} \leq P_{\text {max }, B S} \\
\sum_{k=1}^{K} \log _{2}\left(1+\operatorname{SINR}_{k}\right) \geq R_{\text {min }}
\end{gathered}
$$

The allocated transmission power has been formulated in constraint (12), where each subchannel power have a range within $0-P_{\max , B S}$ and (13) represents the normalized rate requirement.

\section{A. Energy Efficiency Water Filling power allocation algorithm}

In this subsection, we allocate each UE using the Water-Filling algorithm. The overall power allocated to an active user by WF is written as

$$
p_{k}=\left[\frac{1}{g_{k}}-\frac{1}{\mu}\right]_{0}^{P_{\max , B S}}
$$

where the function $[-]_{0}^{P_{\max , B S}}$ confines the range of $p_{k}$ as per (14). Using (3), the SINR of ZF can be written as follows

$$
\operatorname{SINR}_{k}=\frac{p_{k}}{\sigma^{2}+\sum_{k=1}^{K} P_{k}\left|h_{k, i} w_{k, i}\right|^{2}}
$$

the achievable rate for UT $k$ is given by

$$
R_{k}=B \log _{2}\left(1+\operatorname{SINR}_{k}\right)
$$

Hence, the EE is expressed as below

$$
E E=\frac{R_{\text {sum }}}{P_{c}+K p_{k}}
$$

Finally, the EE WF power allocation algorithm is shown in Fig. 2.

Input: $P_{\max , B S}, R_{\min }$
Output: $P, p_{k}, E E$
1: Initialization: $\operatorname{Set} P=P_{\max , B S}$
$\frac{\text { 2: Calculate power allocated: }}{\text { 2.1) Calculate } \mathrm{WF}(P)}$
2.2) Calculate $P_{k}$ in (14)
3: Calculate the minimum rate:
3.1) If $R_{k} \geq R_{\min }$, go to 4.2$)$. If not, continue
3.2) If $p_{k}>P_{\max , B S}$, the problem is unworkable. If not,
calculate $R_{\max }=\log _{2}\left(1+g_{k} P_{\max , B S}\right)$.If
$\quad R_{\min }>\sum_{k=1}^{K} R_{\max }$, the problem is unworkable.
4: Calculate the total power:
4.1) If $p_{k}>P_{\max , B S}$, the problem is unworkable. If not,
calculate EE and exit
4.2) If $p_{k} \leq P_{\max , B S}$, Calculate EE and exit. If not, calculate WF
( $P$ ) and $R_{k}$.
4.3) If $R_{k}<R_{\min }$, the problem is unworkable. If not, calculate EE
and exit.

Fig. 2. Algorithm 1: Energy Efficient WF Power Allocation Algorithm [20]

\section{B. Energy Efficiency proposed power allocation algorithm}

In the following, a proposed power allocation is developed based on an improved water filling (IWF) algorithm by extending the idea of authors [22] to solve the EE optimization problems. Contrary to [22], our proposed power allocation produces simpler structure and allows the differentiability of the system rate function towards the total of power transmitted. Based on the above WF algorithm, an IWF power allocation algorithm is introduced by using the Lagrange Multiplier technique as below 


$$
\forall(P, \lambda, \mu)=\sum_{k=1}^{K} \log _{2}\left(1+\operatorname{SINR}_{k}\right)+\sum_{k=1}^{K} \lambda\left(p_{k}-P_{\max , B S}\right)+\mu\left(\sum_{k=1}^{K} p_{k}-P_{\max , B S}\right)
$$

the vector of the Lagrange Multipliers represents $\mu$ and $\lambda$, respectively. From equation (17), we formulate following optimization problem, aiming to maximize the $\mathrm{EE}$ with constraint (12). An IWF power allocation algorithm is proposed by using the maximum of total squared weights among all the $\mathrm{BSs}$ is given by

$$
S_{k}=\max _{n=1, \ldots, N}\left(\left|w_{k}^{n}\right|^{2}\right)
$$

Therefore,

$$
\begin{aligned}
& \max \left\{\sum_{k=1}^{K} v_{k} *\left(\log _{2}\left(1+g_{k} p_{k}\right)\right)\right\} \\
& \text { subject to } \\
& \sum_{k=1}^{K} p_{k} S_{k} \leq P_{\max , B S}
\end{aligned}
$$

By introducing the Lagrange Multiplier technique, the IWF is defined as below.

$$
\sum_{k=1}^{K} v_{k} * \log _{2}\left(1+g_{k} p_{k}\right)+\mu\left(\sum_{k=1}^{K} p_{k} S_{k}-P_{\max , B S}\right)
$$

Hence, the new optimal power allocated can be written as follows:

$$
p_{k}^{*}=\left[\frac{v_{k}(1+\lambda) B}{(1+\mu) K \ln (2)}-\frac{K}{N-K}\right]_{0}^{P_{\max , B S}}
$$

where $[-]_{0}^{P_{\max , B S}}$ represents the maximum between zero and the $P_{\max , B S}$, while $v_{k} \in[0,1]$ represents each user priority. In a certain case of equal priority, $v_{k}=1 / K$, for all $k$. This corresponds to an IWF distribution with variable water levels that can be changed only by the user priorities. Using (5), the SINR of user can be obtained as:

$$
\operatorname{SINR}_{k}=\frac{g_{k} p_{k}^{*}}{\sigma^{2}}
$$

The pseudo-code for an IWF is summarized in Fig. 3.

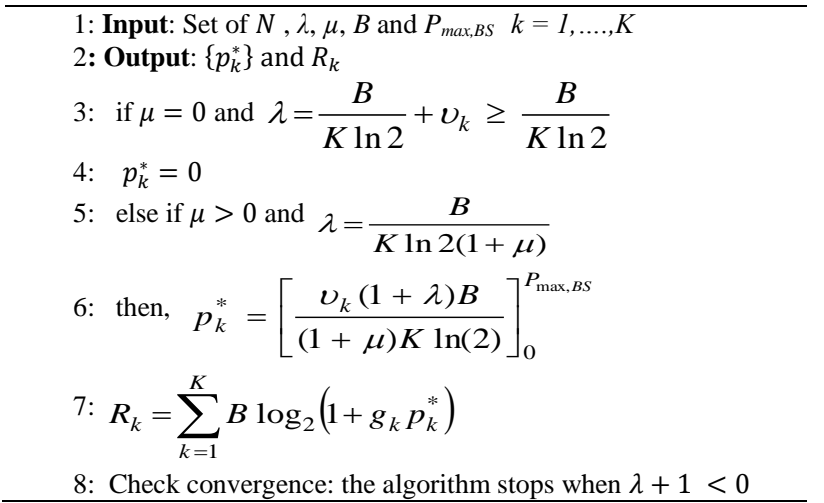

$$
\text { Fig. 3. Algorithm IWF }
$$

The BS allocates each UT with different amounts of power and the power allocation should be allocated. The SINR of UT $k$ is given by

$$
\operatorname{SINR}_{k}=g_{k} p_{k}^{*}
$$

where $p_{k}^{*}$ is the transmit power for UT $k$. Then, we can obtain the value of SINR and the achievable rate for UT $k$ as below

$$
\begin{gathered}
\operatorname{SINR}_{k}^{Z F}=\frac{p_{k}^{*}}{\operatorname{tr}\left(\left(H^{H} H\right)^{-1}\right)} \\
R_{k}=B \sum_{k=1}^{K} \log _{2}\left(1+g_{k} p_{k}^{*}\right)
\end{gathered}
$$

Let $g_{k}$ is received signal to noise ratio. Then, the optimization problem is formulated as follows

$$
\max _{p_{k}^{*}} \frac{\sum_{k=1}^{K} R_{k}\left(p_{k}^{*}\right)}{P_{c x}\left(\sum_{k=1}^{K} \frac{p_{k}^{*}}{\eta}+P_{u c}\right)}
$$

Subject to

$$
\begin{aligned}
& R_{k}\left(p_{k}^{*}\right) \geq R_{\min } \\
& \sum_{k=1}^{K} p_{k}^{*} \leq P_{\max , B S}
\end{aligned}
$$

Based on the above IWF algorithm, we can propose an EE power allocation algorithm by introducing the Lagrange Multiplier technique is defined as below

$$
\begin{aligned}
& \forall(P, \lambda, \mu)=\sum_{k=1}^{K} R_{k}\left(p_{k}^{*}\right)-E E_{k}(P)\left(P_{c x}+\sum_{k=1}^{K} p_{k}^{*}+P_{u c}\right) \\
& +\sum_{k=1}^{K} \lambda\left(R_{k}\left(p_{k}^{*}\right)-R_{\min }\right)-\mu\left(\sum_{k=1}^{K} p_{k}^{*}-P_{\max , B S}\right)
\end{aligned}
$$

Hence, the EE power allocated can be written as follows:

$$
p_{E E}=\left[\frac{v_{k}(1+\lambda) B}{\left(E E_{k}+\mu\right) K \ln (2)}-\frac{K}{N-K}\right]_{0}^{P_{\max , B S}}
$$

The proposed power allocation scheme is more proper and can bring considerable gains over the equal power allocation. The EE an IWF algorithm as shown below.

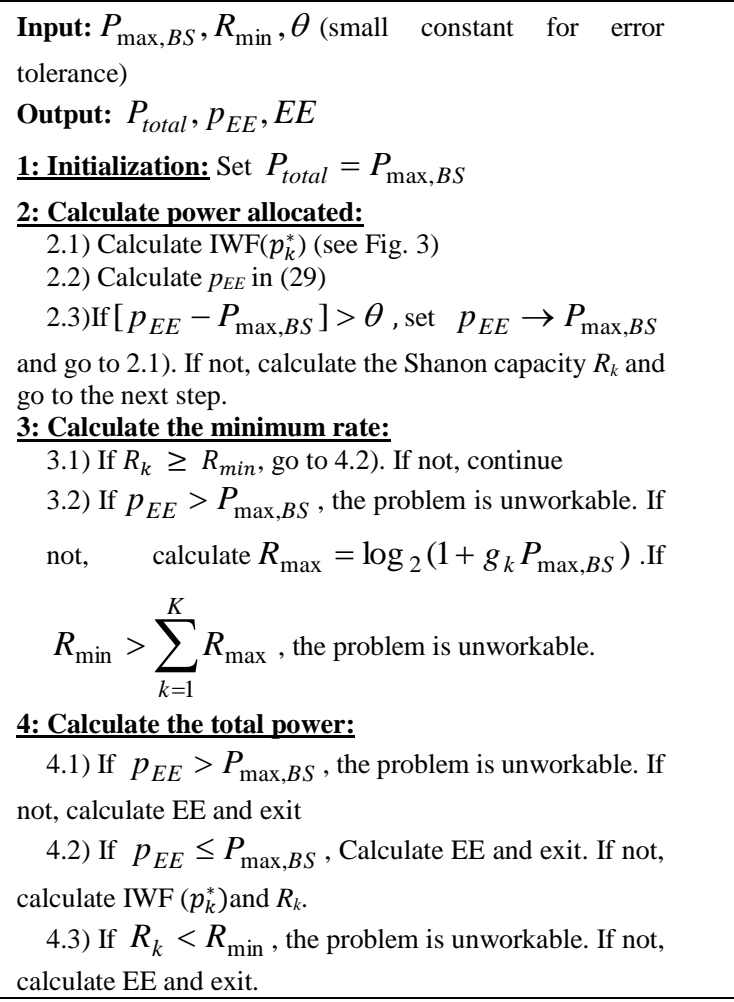

Fig. 4. Energy efficient power allocation with the ZF precoding 


\section{SimUlation AND ANALYSIS}

In this section, we simulate the EE power allocation scheme for Massive MIMO. The proposed power allocation is compared with the existing scheme reported in [16], [20] and [21]. The main parameters used in the simulation are listed in Table II.

Table II

Parameters For The Massive MIMO System [16]

\begin{tabular}{l|l}
\hline Parameter & Value \\
\hline Cell Radius, $R$ & $500 \mathrm{~m}$ \\
System Bandwidth & $20 \mathrm{MHz}$ \\
Circuit power at UT & $0.01 \mathrm{~mW} / \mathrm{Hz}$ \\
Circuit power at BS & $1 \mathrm{~mW} / \mathrm{Hz}$ \\
& \\
Power amplifier efficiency, $\xi$ & 0.5 \\
Path loss exponent, $\alpha$ & 3.7 \\
Noise power spectrum density $\sigma^{2}$ & $-120 \mathrm{dBm} / \mathrm{Hz}$ \\
$\mathrm{R}_{\min } / \mathrm{R}_{\text {th }}$ & $2 \mathrm{Mbps}[21]$ \\
$\mathrm{P}_{\max , \mathrm{BS}}$ & $1.5 \mathrm{~mW}$ \\
\hline
\end{tabular}

Fig. 5 compares the effect of antenna number $N$ with different power allocation algorithm. In this case, increasing $N$ can cause a linear increasing of dynamic and static power. It can be observed that the proposed algorithm outperforms the other schemes [16], [20] and [21]. As seen from this figure, the power allocation algorithm with the ZF, is better than MRT. The reason comes from the effect of the practical dynamic power. Besides, ZF can remove intra-cell interference while maximizing the signal at the desired terminal whereas MRT cannot remove intra-cell interference. In other words, the structure of the Massive MIMO systems has benefited from the use of power efficiently. For that reason, the Massive MIMO systems with a large number of transmit antennas have been developed to transmit data efficiently with low power usage. This verifies the effectiveness of our proposed approach.

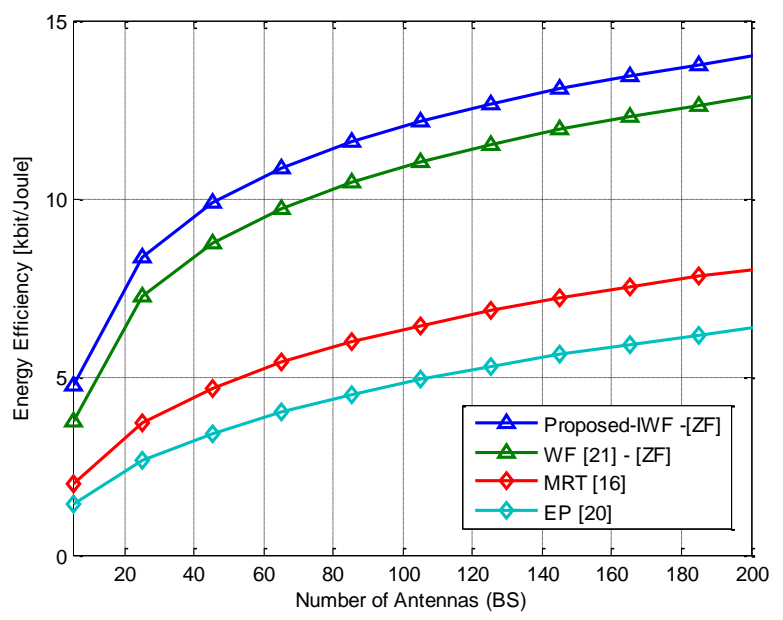

Fig. 5. EE vs the number of antennas at BS

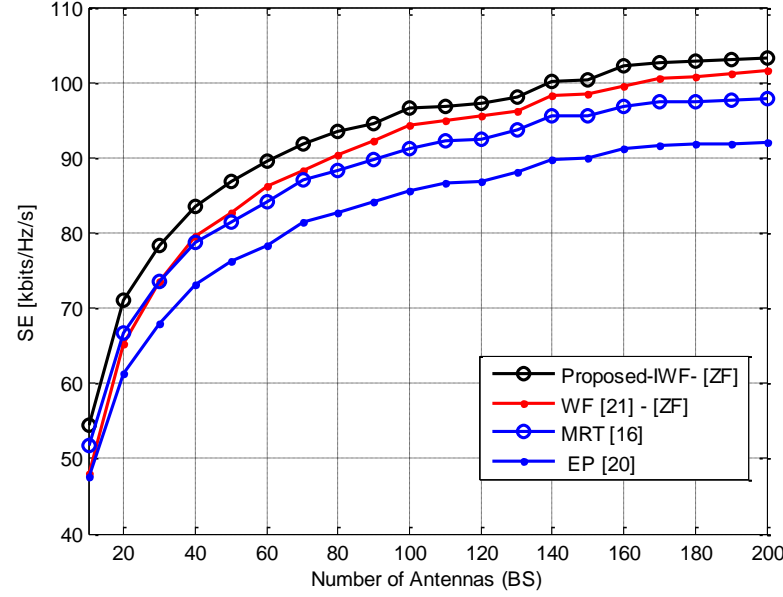

Fig. 6. SE vs the number of antennas at BS

In Fig. 6, SE values of the proposed algorithm are achieved by increasing the number of antennas at BS. The SE performance is reduced when the number of antennas at BS is very small. However, the performance of SE can be improved when the number of users increases and the number of BS antennas is large enough. The inter-cell interference can be cancelled, when using more transmit antennas. So, EE can get the benefits of the existing inter-cell interference at BSs in the downlink communication system with the Massive MIMO systems.

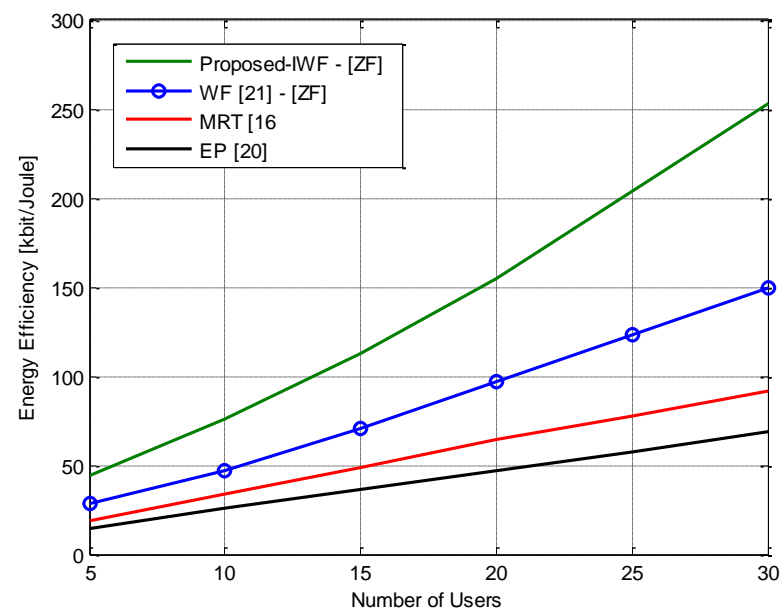

Fig. 7. EE vs the number of users

Fig. 7 compares the optimal EE when the BS has 100 antennas. The number of users is varied to observe its impact on EE. Fig. 7 shows that the performance of EE with the IWF algorithm is better compared with the existing scheme. The optimal EE increases with the increasing of a number of users. This is because more multiuser diversity gain can be obtained by opportunistic scheduling. Besides, the number of users $K$ and transmit antenna number $N$ affect the EE in a complicated manner, adjusting these parameters adaptively is important for improving the EE. 


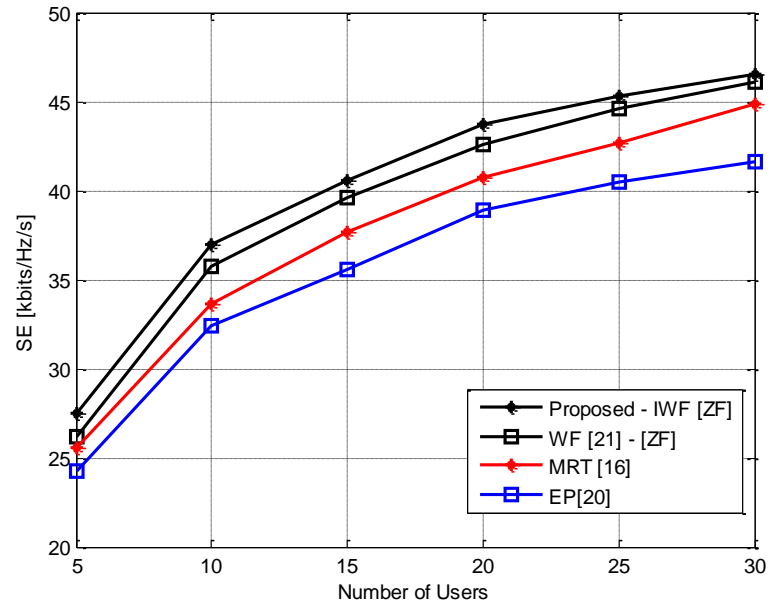

Fig. 8. SE vs the number of users

In Fig. 8, we compare the SE as a function of the number of users. The results indicate that an IWF algorithm is again really effective for enhancing the SE. In our proposed scheme, SE increases as the number of users increases due to the obtainable various multiuser diversity gain. That means when the BSs support increasing number of users, a higher number of antennas BSs are activated, causing the interference and increment of network power consumption. By activating the power allocation strategy, the SINR and average throughput can be increased while reducing the BS power. Therefore, the systems can achieve maximum SE when serving a higher number of users and the number of antennas at MS. It can be observed that by using power allocation strategy, the system performance has improved not only EE but also SE due to the reduction of the cell interference.

\section{CONCLUSION}

In this paper, the energy-efficient power allocation for Massive MIMO was investigated. The energy efficient power allocation was designed based on the IWF algorithm with ZF precoding. The simulation results shown that the IWF outperforms the previous studies by achieving the maximum EE performance. At the same time, the performance of SE also can be improved with Massive MIMO. Based on the proposed power allocation algorithm, we also simulate and analyze the tendency of the EE with the different number of antennas at BS, MS and the number of users.

\section{REFERENCES}

[1] V. Jungnickel, K. Manolakis, W. Zirwas, B. Panzner, V. Braun, M. Lossow, M. Sternad, R. Apelfrojd, and T. Svensson, "The role of small cells, coordinated multipoint, and Massive MIMO in 5G". IEEE Communications Magazine, vol. 52, no. 2, pp. 44-51, 2014

[2] Mohammed H. Alsharif, Rosdiadee Nordin, "Evolution Towards Fifth Generation (5G) Wireless Networks: Current Trends and Challenges in the Deployment of Millimetre Wave, Massive MIMO, and Small Cells", Telecommunication Systems, pp, 1-21, 2016
[3] F. Rusek, Daniel Persson, Buon Kiong Lau, et al., "Scaling Up MIMO: Opportunities and Challenges with Very Large Arrays", IEEE Signal Processing Magazine, vol. 30, no. 1, pp. 40-60, 2013.

[4] D. Feng, C. Jiang, G. Lim, L. J. Cimini, G. Feng, and G. Y. Li, "A Survey of Energy-Efficient Wireless Communications", IEEE Communications Surveys and Tutorials, vol. 15, no. 1,pp. 167-178, 2012.

[5] T.L. Marzetta, "Non cooperative Cellular Wireless with Unlimited Numbers of Base Station Antennas", IEEE Transactions on Wireless Communications, vol. 9 , no. 11 , pp. 3590-3600, 2010 .

[6] J. Hoydis, S. ten Brink, and M. Debbah, "Massive MIMO in the UL/DL of Cellular Networks: How Many Antennas Do We Need?", IEEE Journal on Selected Areas in Communications, vol. 31, no. 2, pp. 160-170, 2013.

[7] H. Q. Ngo, E. G. Larsson, and T. L. Marzetta, "Energy and spectral efficiency of very large multiuser MIMO systems", IEEE Transactions on Communications, vol. 61, no. 4, pp. 1436-1449, 2013.

[8] E. G. Larsson, O. Edfors, and T. L. Marzetta, "Massive MIMO for next generation wireless systems," IEEE Communications Magazine, vol. 52, no. 2, pp. 186-195, 2014.

[9] E. Bjornson, L. Sanguinetti, J. Hoydis, and M. Debbah, "Designing multiuser MIMO for energy efficiency: When is massive MIMO the answer?", In Proceedings IEEE Wireless Communications and Networking Conference (WCNC), Istanbul, 2014.

[10] D. Ng, E. Lo, and R. Schober, "Energy-efficient resource allocation in OFDMA systems with large numbers of base station antennas", IEEE Transactions on Wireless Communications, vol. 11, no. 9, pp. 3292-3304, 2012.

[11] S. K. Mohammed, "Impact of transceiver power consumption on the energy efficiency of zero-forcing detector in massive MIMO systems", IEEE Transactions on Communications, vol. 62, no. 11, pp. 3874-3890, 2014.

[12] Chunlong He, Bin Sheng, Pengcheng Zhu and Xiaohu You, "Energy Efficiency and Spectral Efficiency Tradeoff in Downlink Distributed Antenna Systems", IEEE Wireless Communications Letters, vol. 1, no. 3, pp. 153-156, 2012.

[13] Z. Xu, S. Han, Z. Pan, and C.-L. Yi, "EE-SE relationship for large-scale antenna systems", in Proceedings IEEE International Conference Communications Workshops (ICC), Sydney, 2014.

[14] Y. Hu, Y. M. Huang, and L. X. Yang, "Energy-Efficient Resource Allocation in Multi-user OFDMA systems", International Conference on Wireless Communications and Signal Processing, (WCSP), Nanjing, 2011.

[15] G. Y. Li, Z. Xu, C. Xiong, C. Yang, S. Zhang, Y. Chen, and S. Xu, "Energy-Efficient wireless communications: tutorial, survey, and open issues", IEEE Wireless Communications, vol. 18, no. 6, pp. 28-34, 2011

[16] Long Zhao, Hui Zhao, Kan Zheng and Jingxing Zhang, "Energy Efficient Power Allocation Algorithm for Downlink Massive MIMO with MRT Precoding", IEEE Vehicular Technology Conference (VTC Fall), Las Vegas, 2013.

[17] Yuan Zhou, Dan Li, Haoyu Wang, Ang Yang, Shaozhen Guo, "QoSAware Energy-Efficient Optimization for Massive MIMO Systems in 5G", Sixth International Conference on Wireless Communications and Signal Processing (WCSP), Hefei, 2014

[18] J. Joung, Y. K. Chia and S. Sun, "Energy-efficient, large-scale distributed antenna system (L-DAS) for multiple users", IEEE Journal of Selected Topics in Signal Processing, vol. 8, no. 5, pp. 1932-4553, 2014.

[19] E.Björnson, Sanguinetti, Hoydis, and Debbah, (2014). "Optimal design of energy-efficient multi-user MIMO systems: Is massive MIMO the answer?", IEEE Trans. Wireless Communications, 2014.

[20] Liu, Han, and Yang, "Is massive MIMO energy efficient?" submitted to Trans. on Wireless Communications, 2015.

[21] Z.Zheng, L. Dan, S. Gong and S. Li, "Energy-efficient Resource Allocation for Downlink OFDMA Systems", IEEE International Conference on Communications Workshops (ICC), Budapest, 2013.

[22] Noor Shahida M.K, Rosdiadee Nordin, Mahamod Ismail, "Power Allocation for Dynamic Fractional Frequency Reuse (DFFR) in Downlink LTE-A System", in Proceedings Asia-Pacific Conference on Communications (APCC), Kyoto, Japan, 2015. 